TITLE:

\title{
Numerical study of singularity formation in a class of Euler and Navier-Stokes flows
}

\author{
$\operatorname{AUTHOR}(\mathrm{S}):$
}

Ohkitani, K; Gibbon, JD

\section{CITATION:}

Ohkitani, K ... [et al]. Numerical study of singularity formation in a class of Euler and Navier-Stokes flows. PHYSICS OF FLUIDS 2000, 12(12): 3181-3194

ISSUE DATE:

2000-12

URL:

http://hdl.handle.net/2433/50500

\section{RIGHT:}

Copyright 2000 American Institute of Physics. This article may be downloaded for personal use only. Any other use requires prior permission of the author and the American Institute of Physics. 


\title{
Numerical study of singularity formation in a class of Euler and Navier-Stokes flows
}

\author{
Koji Ohkitani ${ }^{\text {a) }}$ \\ Research Institute for Mathematical Sciences, Kyoto University, Kyoto 606-8502, Japan \\ John D. Gibbon \\ Department of Mathematics, Imperial College of Science, Technology and Medicine, \\ London SW7 2BZ, United Kingdom
}

(Received 20 September 1999; accepted 15 August 2000)

\begin{abstract}
We study numerically a class of stretched solutions of the three-dimensional Euler and NavierStokes equations identified by Gibbon, Fokas, and Doering (1999). Pseudo-spectral computations of a Euler flow starting from a simple smooth initial condition suggests a breakdown in finite time. Moreover, this singularity apparently persists in the Navier-Stokes case. Independent evidence for the existence of a singularity is given by a Taylor series expansion in time. The mechanism underlying the formation of this singularity is the two-dimensionalization of the vorticity vector under strong compression; that is, the intensification of the azimuthal components associated with the diminishing of the axial component. It is suggested that the hollowing of the vortex accompanying this phenomenon may have some relevance to studies in vortex breakdown. (C) 2000 American Institute of Physics. [S1070-6631(00)00312-3]
\end{abstract}

\section{ISSUES CONCERNING THE INCOMPRESSIBLE THREE-DIMENSIONAL EULER EQUATIONS}

\section{A. Introduction}

The formation of spontaneous singularities in the threedimensional (3D) incompressible Euler equations is one of the outstanding problems in modern fluid dynamics. These equations can be expressed in the usual form

$$
\frac{\partial \boldsymbol{U}}{\partial t}+\boldsymbol{U} \cdot \nabla \boldsymbol{U}=-\nabla p
$$

with the velocity field $\boldsymbol{U}(x, y, z, t)=(u, v, w)$ subject to the incompressibility condition $\operatorname{div} \boldsymbol{U}=0$. Evidence for a singularity in flows of finite energy has been reported in a variety of circumstances. Kerr's numerical integration, ${ }^{1}$ starting from an initial state of a pair of anti-parallel vortex tubes, strongly suggests that the vorticity $\boldsymbol{\Omega}=\operatorname{curl} \boldsymbol{U}$ blows up at a point in a finite time with $\|\boldsymbol{\Omega}\|_{\infty} \sim\left(t^{*}-t\right)^{-1}$. Despite progress in analytical methods, a rigorous proof that a singularity could develop in a finite time from certain classes of initial data has remained an open problem since the time of Leray $^{2}$ who considered the Navier-Stokes case, while numerical results are only suggestive. The result of Beale, Kato, and Majda (BKM), ${ }^{3}$ who showed that no quantity associated with (1) can become singular at a time $t^{*}$ without $\int_{0}^{t^{*}}\|\boldsymbol{\Omega}(\tau)\|_{\infty} d \tau$ also becoming singular at $t^{*}$, has had a major influence on the subject because it gives the computational fluid dynamicist a benchmark against which to test whether a singularity arising from a numerical experiment is real or a numerical artifact. Constantin, Fefferman, and Majda ${ }^{4}$ have more recently refined this result by bringing

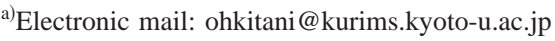

down the $L^{\infty}$-norm in the above time integral to a finite value at the cost of imposing conditions on the direction of vorticity (see also Ref. 5). In a different manner, Ponce ${ }^{6}$ has also shown that the BKM result can be modified in such a way that the $\|\boldsymbol{\Omega}\|_{\infty}$-norm within the time integral is replaced by $\|S\|_{\infty}$, where $S$ is the strain matrix.

An alternative approach is to take advantage of the cost savings gained by using high symmetry initial conditions. ${ }^{7-9}$ Bhattacharjee et al. ${ }^{10}$ (see also Ref. 11) have discussed in some detail how Euler singularities may develop from symmetric initial conditions, such as the imposition of Kida's high-symmetry initial condition. ${ }^{7}$ By using perturbative methods they suggest evidence for two types of possible singular behavior of $\boldsymbol{\Omega}$. Pelz and Gulak ${ }^{12}$ have also used Kida's initial condition to follow the development of a real-time singularity using Padé methods of analysis.

All the flows discussed above are finite in energy. In this paper we study the possibility of singularity formation in solutions of the 3D Euler equations that have imposed upon them an infinite geometric structure in one particular direction. Such flows, being infinite in one distinguished direction, have infinite energy and therefore must be placed in a different category than those discussed in the above references. To be specific, let us consider a class of 3D Euler velocity fields whose only $z$ dependence lies linearly in the third component

$$
\boldsymbol{U}(x, y, z, t)=\left(\begin{array}{c}
u \\
v \\
w
\end{array}\right)=\left(\begin{array}{c}
u(x, y, t) \\
v(x, y, t) \\
z \gamma(x, y, t)+W(x, y, t)
\end{array}\right) .
$$

The vorticity has two components that are linear in $z$ and one independent of $z$ 


$$
\boldsymbol{\Omega}(x, y, z, t)=\left(\begin{array}{c}
\Omega_{1} \\
\Omega_{2} \\
\Omega_{3}
\end{array}\right)=\left(\begin{array}{c}
z \frac{\partial \gamma}{\partial y}+\frac{\partial W}{\partial y} \\
-z \frac{\partial \gamma}{\partial x}-\frac{\partial W}{\partial x} \\
\omega
\end{array}\right),
$$

where

$$
\omega(x, y, t)=\frac{\partial v}{\partial x}-\frac{\partial u}{\partial y}
$$

The strain matrix, $S$, associated with this velocity field is

$$
S=\left(\begin{array}{rrr}
S_{11} & S_{12} & -\frac{1}{2} \Omega_{2} \\
S_{21} & S_{22} & \frac{1}{2} \Omega_{1} \\
-\frac{1}{2} \Omega_{2} & \frac{1}{2} \Omega_{1} & \gamma
\end{array}\right) .
$$

This class of velocity fields is of stagnation point type and, as such, has its limitations. Clearly, it cannot be thought of as representing general 3D Euler flows of finite energy. Nevertheless, it does include several well known and important subclasses of solutions of the Euler equations:

(1) The Burgers vortex is the most prominent member of this class; ${ }^{13}$ take $W=0$ and restrict $\gamma$ to being a function of time only. Writing $(u, v)$ in strain-field form with a stream function $\psi=\psi(x, y, t)$ we have

$u=-\frac{1}{2} \gamma(t) x+\frac{\partial \psi}{\partial y}, \quad v=-\frac{1}{2} \gamma(t) y-\frac{\partial \psi}{\partial x}, \quad w=z \gamma(t)$.

The Euler version of the Burgers vortex can easily be found from this formulation. This vortex, both in its Euler and Navier-Stokes manifestations, is a well-known solution that is thought to represent tubes of vorticity in both inviscid and viscous turbulent flows. ${ }^{14}$ Clearly, if $\gamma$ is chosen to be spatially uniform and $W=0$ then $\Omega_{1}$ $=\Omega_{2}=0$ and the axial component $\omega=-\Delta \psi$ is uniform in the $z$ direction. This uniformity contributes to the infinite tube-like geometry and makes the Burgers vortex an infinite energy solution. When $W \neq 0$, however, a variety of other solutions have recently been shown to exist (see the discussion in Ref. 15).

(2) A solution of the three-dimensional Euler equations that blows up in finite time and has a double stagnation point has been found by Stuart. ${ }^{16}$ His velocity field has a dual linear structure and takes the form $u=x \bar{u}(y, t), v$ $=\bar{v}(y, t)$, and $w=z \gamma(y, t)$. This problem is more amenable to a Lagrangian than an Eulerian formulation, as Stuart has shown, and is appropriate for boundary layer studies. ${ }^{16,17}$ Finite time singularities have long been thought to play a significant role in the separation of boundary layers. The paper by Cowley et al. ${ }^{18}$ reviews the Lagrangian description of this type of problem.

(3) The two-dimensional model by Childress et al. ${ }^{19}$ of blow-up in the two-dimensional (2D) Euler equations belongs to the class of velocity fields (2). Taking $w=0$, their velocity field is of the divergence-free form $(u, v)=\left(u(x, t),-y u_{x}(x, t)\right)$.

This two-dimensional problem has a single stagnation point structure, as opposed to the double point in Stuart's solution, which is three-dimensional and stretches in two directions. ${ }^{16}$ Childress et al. use the phrase "stagnation point similitude" when referring to the linear structure of the distinguished variable. ${ }^{19}$

Finally we add two further examples to the list of Euler solutions that blow up. First, Constantin's “distorted Euler equation" differs from the true three-dimensional Euler equations in that a singular integral operator with a time varying kernel in the equation for the velocity gradient matrix $U_{i, j}$ is replaced by its initial value. ${ }^{20}$ This system develops a finite time singularity for various initial data. Second, Moffatt has recently constructed a singular solution of the 3D Euler equations by generalizing the Burgers vortex. ${ }^{21}$ This solution, whose vorticity blows up at a point, has singular and unbounded straining fields in all three directions and so has infinite energy.

\section{B. Equations of motion for $\gamma, \mathbf{W}$, and $\omega$}

Gibbon, Fokas, and Doering ${ }^{15}$ recently showed that if the class of velocity fields $\boldsymbol{U}$ given in (2) satisfies the 3D Navier-Stokes equations then simple separate equations for $\gamma(x, y, t), W(x, y, t)$ and the third component of vorticity $\omega(x, y, t)$ can be derived; in other words, the $z$ dependence can be removed from the system. With the greatest brevity we repeat this calculation in the Euler context.

Let us define the 2D velocity field part of (2) as

$$
\boldsymbol{u}(x, y, t)=(u(x, y, t), v(x, y, t))
$$

and let $\nabla$ be the 2D gradient operator. Then, from (1), $\boldsymbol{u}$ satisfies

$$
\frac{\partial \boldsymbol{u}}{\partial t}+\boldsymbol{u} \cdot \nabla \boldsymbol{u}=-\nabla p
$$

These are not, however, the true 2D Euler equations because

$$
\operatorname{div} \boldsymbol{u}=-\gamma
$$

We need a pair of evolution equations for $\gamma$ and $W$. The third velocity field $w=z \gamma+W$ gives this information. Recalling that the $3 \mathrm{D}$ total derivative is

$$
\frac{D}{D t}=\frac{\partial}{\partial t}+\boldsymbol{u} \cdot \nabla+w \frac{\partial}{\partial z}
$$

we can easily calculate $\partial p / \partial z$ from the third component of (1)

$$
-\frac{\partial p}{\partial z}=z\left(\frac{\partial \gamma}{\partial t}+\boldsymbol{u} \cdot \nabla \gamma+\gamma^{2}\right)+\left(\frac{\partial W}{\partial t}+\boldsymbol{u} \cdot \nabla W+\gamma W\right) .
$$

On integration with respect to $z$ we have 


$$
\begin{aligned}
-p(x, y, z, t)= & \frac{1}{2} z^{2}\left(\frac{\partial \gamma}{\partial t}+\boldsymbol{u} \cdot \nabla \gamma+\gamma^{2}\right) \\
& +z\left(\frac{\partial W}{\partial t}+\boldsymbol{u} \cdot \nabla W+\gamma W\right)+P(x, y, t),
\end{aligned}
$$

where $P(x, y, t)$ is a function of integration. The stagnation point type structure shows up in a typical way. The crux of the matter lies in the following argument; because Eq. (1) insists that the partial derivatives of the pressure, $\partial p / \partial x$ and $\partial p / \partial y$, are independent of $z$, the only way to prevent a contradiction between this fact and the quadratic $z$ structure in $p$, is to demand that the coefficients of $z$ and $z^{2}$ in (13) are independent of $x$ and $y$, although they can be arbitrary functions of time. The coefficient of $z$ can be put to zero because this represents no more than an accelerating change of frame, but the coefficient of $z^{2}$ has more significance. It means that $p_{z z}$ the second partial $z$ derivative of the pressure, must be a spatially uniform arbitrary function of time; that is, $p_{z z}$ $=p_{z z}(t)$. Hence we have

$$
\begin{aligned}
& \frac{\partial \gamma}{\partial t}+\boldsymbol{u} \cdot \nabla \gamma=-\gamma^{2}-p_{z z}(t), \\
& \frac{\partial W}{\partial t}+\boldsymbol{u} \cdot \nabla W=-\gamma W .
\end{aligned}
$$

These are two of the three equations derived in Ref. 15. To obtain the third we look at the block diagonal structure of the strain matrix $S$ given in (5) and note that the third component of $S \boldsymbol{\Omega}$ decouples such that

$$
(S \Omega)_{3}=\gamma \omega
$$

with $\omega$ defined in (4). The vorticity version of the 3D Euler equations shows that the third equation of the set must be

$$
\frac{\partial \omega}{\partial t}+\boldsymbol{u} \cdot \nabla \omega=\gamma \omega
$$

This is the third of the three equations derived in Ref. 15. Very recently we discovered that in 1927 Oseen $^{22}$ derived a time independent version of these equations with $W=0$.

So far, no domain has been specified. In Ref. 15 it was shown that on a domain infinite in all directions, with $\gamma$ restricted to being spatially uniform, then (17) can be rescaled to the 2D Euler equations with $W$ acting as a passive scalar on that flow. This is a generalization of Lundgren's straining transformation $^{23}$ (see Ref. 15). There $\gamma=\gamma(t)$ behaves as a dynamical variable which affects the system through the scaling variable $s(t)=\exp \left(\int_{0}^{t} \gamma(\tau) d \tau\right)$. Through this transformation any solution of 2D Euler (with an additional passive scalar) on an infinite domain can be mapped back to form a solution of 3D Euler within the class of velocity fields (2). The dynamics of $\gamma$ are, therefore, important but are somewhat arbitrary because the driving function $p_{z z}(t)$ is arbitrary. To determine $\gamma$ requires the specification of some boundary conditions.

\section{The equations in a tube-like domain}

The coupled equations (14), (15), and (17) have the advantage of being functions of only two independent variables, thereby enabling us to more easily undertake a numerical investigation of their properties. The postulated linear $z$ dependence in the full 3D Euler equations distinguishes the $z$ coordinate, so this must be taken into account when choosing boundary conditions. We choose a domain that is unbounded in the $z$ direction but, for simplicity, we assume that every field is periodic in $x, y$ on the finite crosssectional domain $\mathcal{A}=[0,2 \pi]^{2}$. The chosen domain is, therefore, very like an infinite tube with a periodic cross section.

Given these boundary conditions it is now clear that the continuity condition (10)

$$
\gamma=-\operatorname{div} \boldsymbol{u}=-\left(\frac{\partial u}{\partial x}+\frac{\partial v}{\partial y}\right),
$$

tells us that $\gamma$ is a mean-zero function over the crosssectional domain $\mathcal{A}$

$$
\int_{\mathcal{A}} \gamma d \boldsymbol{x}=0
$$

Using this information in (14) fixes $p_{z z}(t)$ to be

$$
p_{z z}(t)=-2\left\langle\gamma^{2}\right\rangle=-2 \frac{1}{(2 \pi)^{2}} \int_{\mathcal{A}} \gamma^{2} d x,
$$

where the bracket $\langle\cdot\rangle$ stands for the square of the $L^{2}$-spatial average of $\gamma$. Hence our three equations of motion for $\gamma, W$ and $\omega$ are now

$$
\begin{aligned}
& \frac{\partial \gamma}{\partial t}+\boldsymbol{u} \cdot \nabla \gamma=-\gamma^{2}+2\left\langle\gamma^{2}\right\rangle, \\
& \frac{\partial W}{\partial t}+\boldsymbol{u} \cdot \nabla W=-\gamma W, \\
& \frac{\partial \omega}{\partial t}+\boldsymbol{u} \cdot \nabla \omega=\gamma \omega,
\end{aligned}
$$

together with (18). From its definition in (4), $\omega$ can be written as $\hat{\boldsymbol{k}} \omega=\operatorname{curl} \boldsymbol{u}$, where $\hat{\boldsymbol{k}}$ is a unit vector in $z$ direction. This allows a Helmholtz-Hodge decomposition to be used to solve for $u$ and $v$. This decomposes the vector field $\boldsymbol{u}$ into irrotational and solenoidal parts

$$
\boldsymbol{u}=-\nabla\left(\Delta^{-1} \gamma\right)-\operatorname{curl}\left(\hat{\boldsymbol{k}} \Delta^{-1} \omega\right) .
$$

Hence the velocity components $u$ and $v$ are solely determined by $\gamma$ and $\omega$ through the inverse two-dimensional Laplacian $\Delta^{-1}$ as follows:

$$
u=-\Delta^{-1}\left(\frac{\partial \omega}{\partial y}+\frac{\partial \gamma}{\partial x}\right), \quad v=\Delta^{-1}\left(\frac{\partial \omega}{\partial x}-\frac{\partial \gamma}{\partial y}\right) .
$$

Clearly the other variable $W$ plays no part in this determination, having only a passive role.

We solve Eqs. (21)-(23) numerically using a standard pseudo-spectral method in double precision arithmetic. The aliasing errors are suppressed by the 2/3-law and the number of effective degrees of freedom is $(2 N / 3)^{2}$ for a computation 
of $N^{2}$ grid points. The time marching is performed by the fourth-order Runge-Kutta method with use of a typical time increment $\Delta t=10^{-3}$. We have checked that the properties of the solutions do not change by increasing resolution as $N$ is increased from 256 to 512 and up to 1024 and 2048 in some cases. We will typically use $N=256$. In contrast to solutions of the usual 2D and 3D Euler equations, a rather low resolution is sufficient to resolve the flow field. The reason for this is that the Fourier spectrum of the energy keeps to an exponential fall-off close to the end of the computation (see below).

Clearly the key variable in the system is $\gamma$; the main issue in this paper is whether $\gamma$ blows up in finite time and, if so, what the nature of this blow-up is. The variable $W$, and to a lesser degree $\omega$, can be thought of as subsidiary variables; $\gamma$ controls the growth or decay of $W$ and $\omega$ depending on whether its growth is positive or negative. Blow-up in $\gamma$ means blow-up in at least the first two components of the vorticity vector $\boldsymbol{\Omega}$ and in several components of the strain matrix $S$. Blow-up in $\omega$ depends on the sign of $\gamma$ as it blows up.

\section{THE INVISCID PROBLEM}

We consider whether smooth solutions of (21)-(23) persist or not, beginning with the following naive observation. If we neglect the second term on the right-hand-side (rhs) of Eq. (21) for $\gamma$, we find

$$
\frac{\partial \gamma}{\partial t}+\boldsymbol{u} \cdot \nabla \gamma=-\gamma^{2}
$$

This can be trivially solved in a Lagrangian frame and for some fluid particles $\gamma$ tends to $-\infty$ in finite time. But, since the second term on the rhs of (21) is positive and can become large, it might prevent formation of singularity. Because of this competition, it is not obvious that solutions of (21)-(23) break down in finite time or not. Therefore, we need to study the problem numerically.

\section{A. Initial condition 1}

As there are no guidelines for choosing appropriate initial conditions, we first try a simple sinusoidal one for all the three fields:

$$
\omega(\boldsymbol{x}, 0)=\gamma(\boldsymbol{x}, 0)=W(\boldsymbol{x}, 0)=\sin x \sin y .
$$

Unlike 2D Euler equations, a time dependent solution arises from this single mode initial condition.

We describe the global properties of the flow using several norms. Denoting the spatial average by the brackets [see (20)], we define energy-like norms

$$
\begin{aligned}
& E_{\omega}(t)=\frac{1}{2}\left\langle\left((-\Delta)^{-1 / 2} \omega(\boldsymbol{x}, t)\right)^{2}\right\rangle, \\
& E_{\gamma}(t)=\frac{1}{2}\left\langle\left((-\Delta)^{-1 / 2} \gamma(\boldsymbol{x}, t)\right)^{2}\right\rangle, \\
& E_{W}(t)=\frac{1}{2}\left\langle\left((-\Delta)^{-1 / 2} W(\boldsymbol{x}, t)\right)^{2}\right\rangle
\end{aligned}
$$

and enstrophy-like norms

$$
Q_{\omega}(t)=\frac{1}{2}\left\langle\omega(\boldsymbol{x}, t)^{2}\right\rangle,
$$



(a)

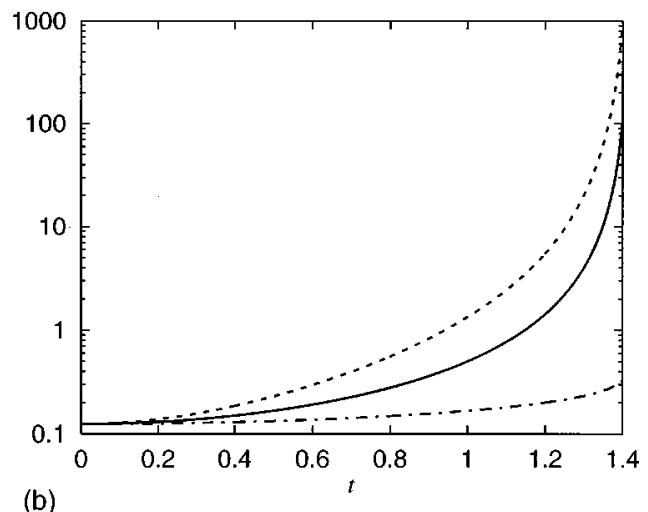

FIG. 1. Time evolution of the norms for the initial condition 1; (a) $E_{\omega}(t)$ (dash-dotted), $E_{\gamma}(t)$ (solid), and $E_{W}(t)$ (dashed) and (b) $Q_{\omega}(t)$ (dashdotted), $Q_{\gamma}(t)$ (solid), and $Q_{W}(t)$ (dashed).

$$
\begin{aligned}
& Q_{\gamma}(t)=\frac{1}{2}\left\langle\gamma(\boldsymbol{x}, t)^{2}\right\rangle, \\
& Q_{W}(t)=\frac{1}{2}\left\langle W(\boldsymbol{x}, t)^{2}\right\rangle,
\end{aligned}
$$

with a slight abuse of terminology for $Q_{W}$ because $W$ has dimension of velocity. None of these are conserved in time.

We show the time evolution of $E_{\omega}(t), E_{\gamma}(t)$, and $E_{W}(t)$ in Fig. 1(a). From this we see that $E_{\omega}(t)$ does not become large (actually it decreases slightly) but $E_{\gamma}(t)$ and $E_{W}(t)$ indeed increase rapidly and they appear to diverge at about $t=1.4$, or more precisely $t=1.419$. A similar trend is observed in Fig. 1(b), which shows evolution of $Q_{\omega}(t), Q_{\gamma}(t)$, and $Q_{W}(t)$. Again, $Q_{\gamma}(t)$ and $Q_{W}(t)$ appear to diverge at about the same time $t=1.4$ but $Q_{\omega}(t)$ increases only by a small amount.

By changing the resolution, we found that $Q_{\gamma}(t)$ diverges at $t=1.419$ with $N^{2}=128^{2}$ and $256^{2}, t=1.414$ with $512^{2}, t=1.396$ with $1024^{2}, t=1.349$ with $2048^{2}$. The difference in the time of numerical blow up is small, that is, $3 \%$. As long as the computation is possible, the curves of $Q_{\gamma}(t)$ collapse completely. A possible reason for slightly earlier breakdown of the numerical solutions at higher resolutions is that they have less numerical dissipation.

Under the periodic boundary condition, we have

$$
\langle\omega\rangle=0 \text {, }
$$

by (4). We have checked this together with the condition $\langle\gamma\rangle=0$ in the computation. On the other hand, $\langle W\rangle$ is not conserved 


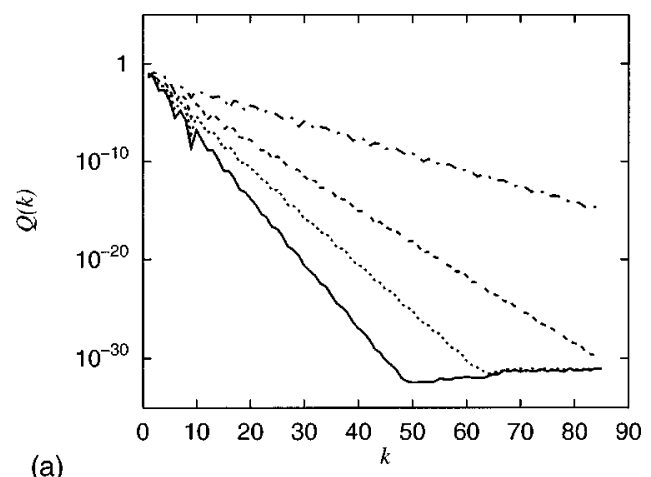

(a)
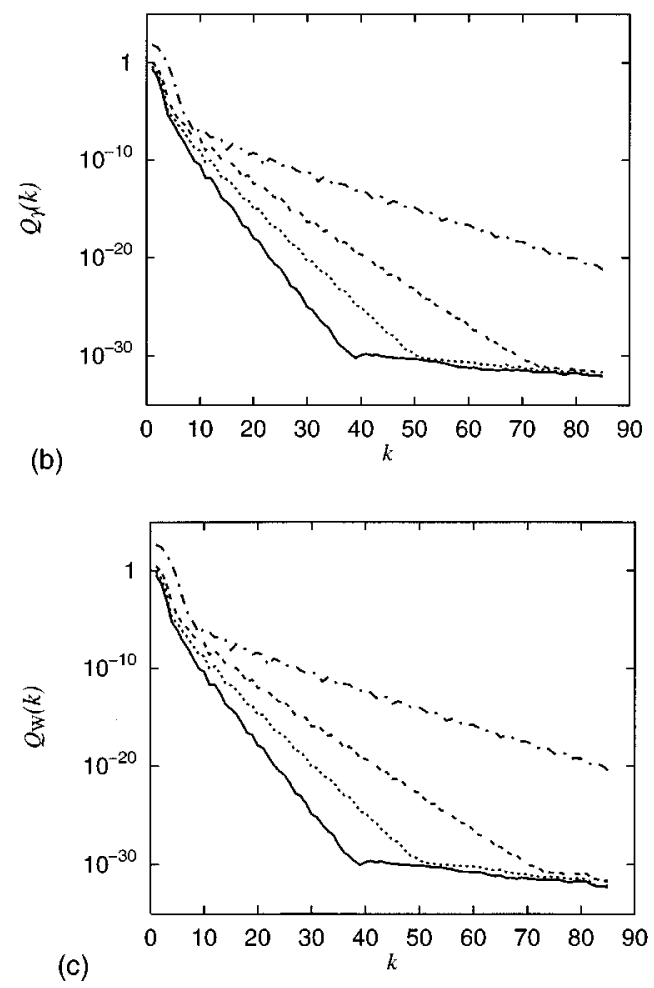

$$
\frac{d}{d t}\langle W\rangle=-2\langle\gamma W\rangle
$$

Actually, $\langle W\rangle$ also appears to diverge to $-\infty$ at $t=1.4$ (figure omitted). This means that the maximum value of $|W|$ also diverges in finite time. It should be noted, therefore, that not only the velocity gradient but also the velocity itself appear to become singular in finite time.

When we perform computation of the problems which may lead to the formation of singularity, we need to take special care so that the numerical solutions are sufficiently well resolved. To this end we monitor Fourier spectra of the three fields defined by

$$
\begin{aligned}
& Q_{\omega}(k)=\frac{1}{2} \sum_{k \leqslant|k|<k+1}|\tilde{\omega}(\boldsymbol{k})|^{2}, \\
& Q_{\gamma}(k)=\frac{1}{2} \sum_{k \leqslant|k|<k+1}|\tilde{\gamma}(\boldsymbol{k})|^{2},
\end{aligned}
$$

and
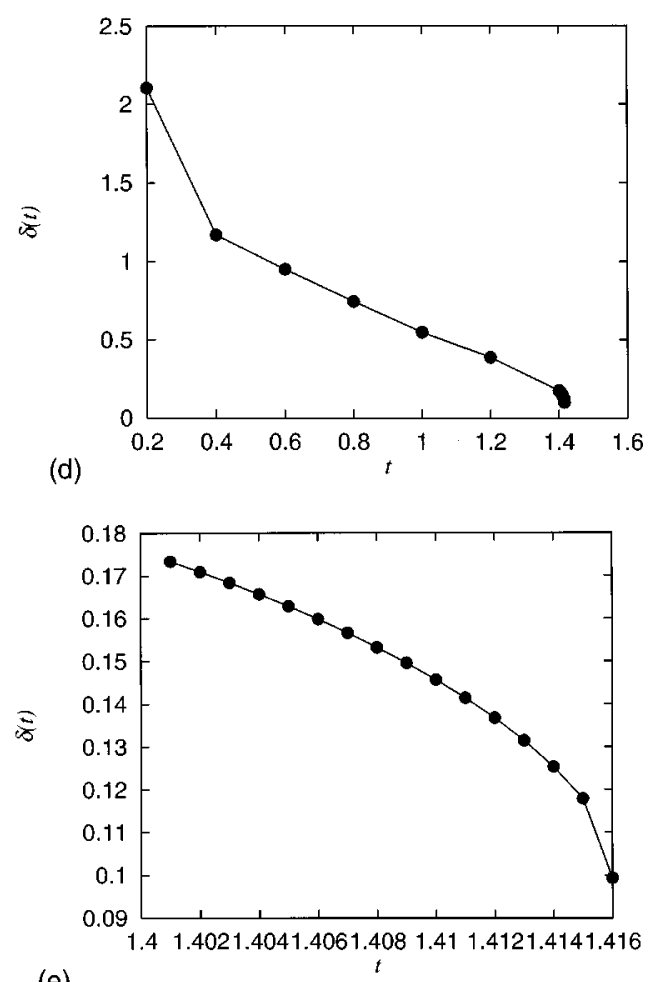

(e)

FIG. 2. Time evolution of the Fourier spectra for the initial condition 1; (a) $Q_{\omega}(k)$, (b) $Q_{\gamma}(k)$, and (c) $Q_{W}(k)$; at $t=0.8$ (solid), 1.0 (dotted), 1.2 (dashed), 1.4 (dash-dotted), (d) the analyticity distance $\delta(t)$, and (e) its details.

$$
Q_{W}(k)=\frac{1}{2} \sum_{k \leqslant|k|<k+1}|\widetilde{W}(\boldsymbol{k})|^{2} .
$$

We show log-linear plots of $Q_{\omega}(k)$ in Fig. 2(a) at $t$ $=0.8,1.0,1.2$, and 1.4. It should be noted that the straight lines in the higher wave number range imply exponential fall-offs. This suggests that the flow is well-resolved close to the end of the computation. We can also confirm that $\gamma$ and $W$ are similarly well resolved by observing Figs. 2(b) and 2(c).

To study the evolution of the Fourier spectrum in more detail, we consider $Q_{\gamma}(k)$ and fit it in the usual form as

$$
Q_{\gamma}(k)=A(t) k^{n(t)} \exp (-2 \delta(t) k)
$$

where the constants $A(t), n(t), \delta(t)$ are determined by the least-squares method at each time (see, for example, Ref. $24)$. The distance $\delta(t)$ is a measure of analyticity of the 




(a)

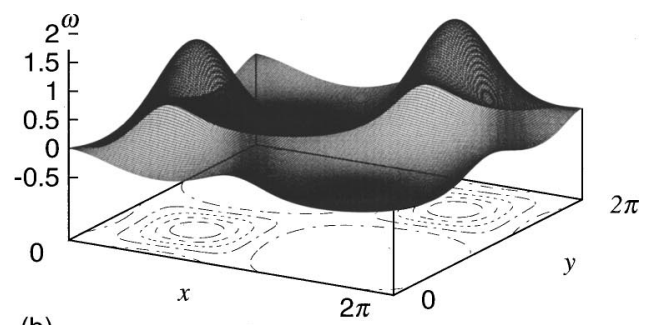

(b)

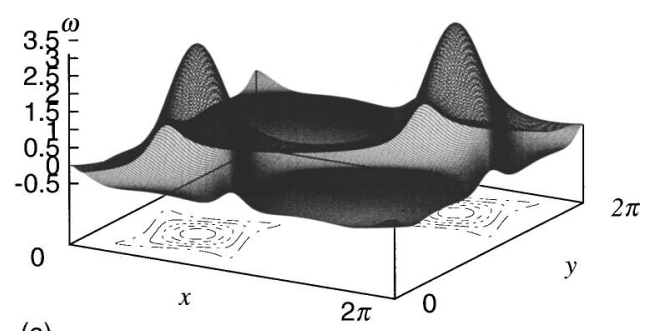

(c)

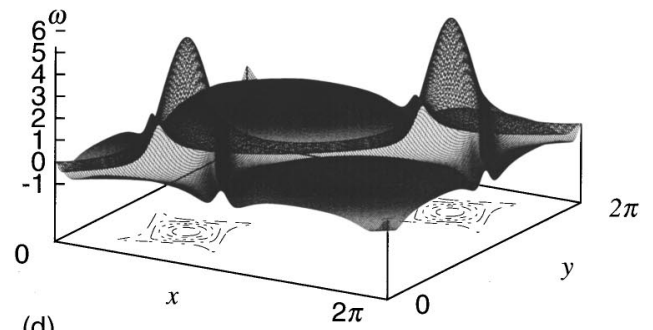

(d)

FIG. 3. Perspective plots and contours of $\omega$ for the initial condition 1; (a) $t=0$, (b) $t=0.6$, (c) $t=1.2$, and (d) $t=1.4$.

function $\gamma(\boldsymbol{x})$. We plot in Fig. 2(d) the time evolution of $\delta(t)$ for the computation with $N=256$, where the fitting procedure was performed in the wave number range $k \geqslant 10$, excluding the round-off noise. Up to $t=1.4, \delta(t)$ is much larger than the mesh size $2 \pi / N \approx 0.025$, which is a necessary condition for the flow to be sufficiently well resolved. But after $t=1.4$, the analyticity distance appears to decrease much faster. Such a behavior is confirmed in Fig. 2(e), where we show a close up view of $\delta(t)$ as a function of $t$ in 1.4 $\leqslant t \leqslant 1.416$. We have checked that the decrease of $\delta(t)$ is faster than an exponential function in time. The tail part of the Fourier spectra begins to turn up at $t=1.417$ (not shown), after this time the computation becomes inaccurate. The fast decrease of $\delta(t)$ suggests its vanishing near $\mathrm{t}=1.416$, that is, a blowup.

We have also performed computations by varying the time step $\Delta t$ for $N=1024$. The blowup times are $t=1.396$ for $\Delta t=1 \times 10^{-3}, t=1.408$ for $\Delta t=5.0 \times 10^{-4}, t=1.413$ for $\Delta t=2.5 \times 10^{-4}$. Our conclusion is that at this high resolution



(a)



(b)
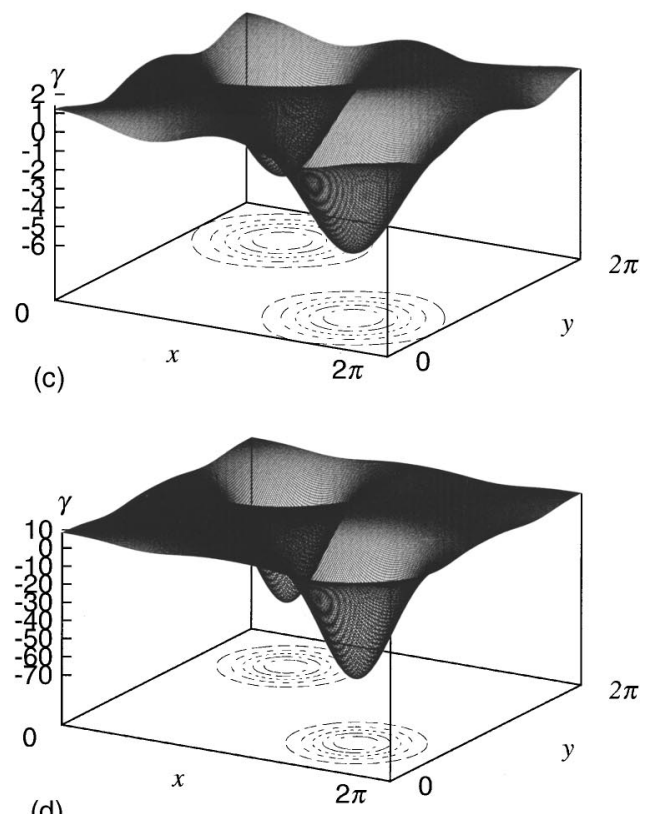

(d)

FIG. 4. Perspective plots and contours of $\gamma$ for the initial condition 1; (a) $t=0$, (b) $t=0.6$, (c) $t=1.2$, and (d) $t=1.4$.

of $N=1024$ the time of numerical breakdown appears to be near $t=1.4$. This indicates the time scale becomes increasingly small, consistent with a real blowup (see the note added in proof).

Next we study the spatial structure on the twodimensional periodic domain $\mathcal{A}$. In Fig. 3 we show perspective plots of $\omega$ at $t=0,0.6,1.2$, and 1.4. This initial condition has four cells. In the subregions where $\omega$ is positive this variable undergoes a mild increase and, by the end of computation, its maximum is larger than the initial maximum approximately by a factor of 6 . Keeping in mind that initially $\omega=\gamma$, this comes from stretching by positive strain rate $\gamma$. In other subregions, the strain rate $\gamma$ is initially negative, and accordingly $|\omega|$ is getting smaller and smaller. At later times we see a large almost flat area. In either region, the growth of $\omega$ is not significant. 


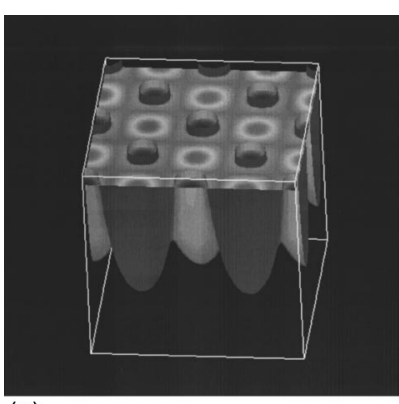

(a)

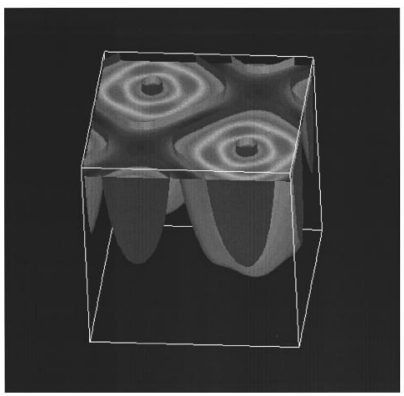

(b)

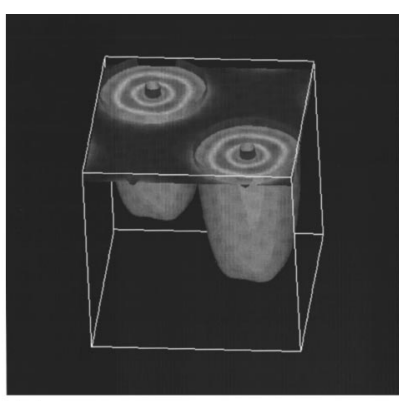

(c)

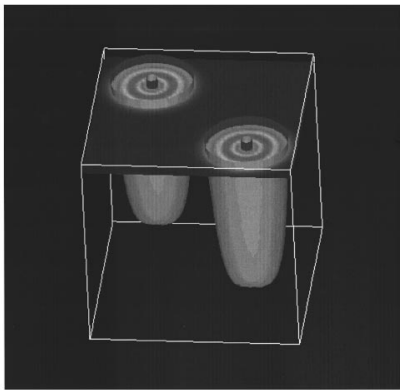

(d)

FIG. 6. Three-dimensional enstrophy for the initial condition 1; (a) $t=0$, (b) $t=0.6$, (c) $t=1.2$, and (d) $t=1.4$. Its distribution of the sectional plane is also shown, where darker shading denotes higher values of enstrophy.

$$
|\boldsymbol{\Omega}(x, y, z, t)|^{2}=\left(z \frac{\partial \gamma}{\partial y}+\frac{\partial W}{\partial y}\right)^{2}+\left(z \frac{\partial \gamma}{\partial x}+\frac{\partial W}{\partial x}\right)^{2}+\omega(\boldsymbol{x})^{2}
$$

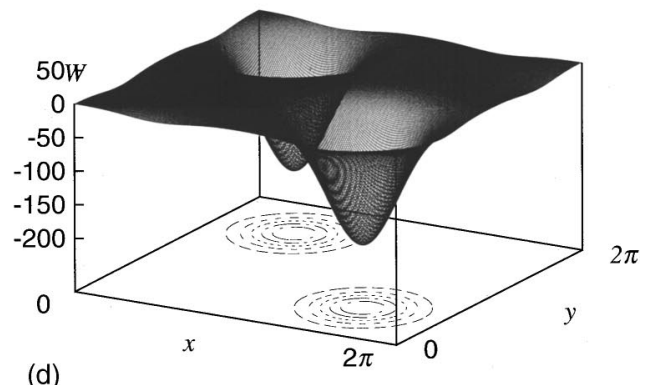

(d)

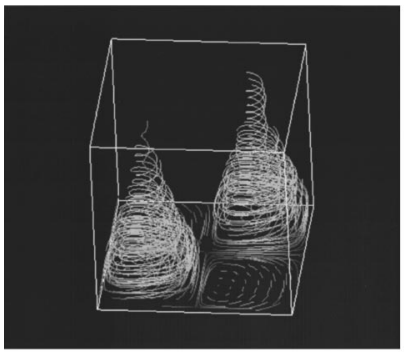

(a)

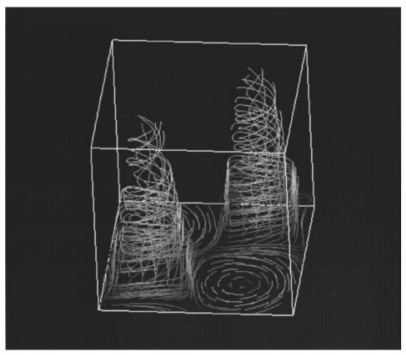

(b)



(c)

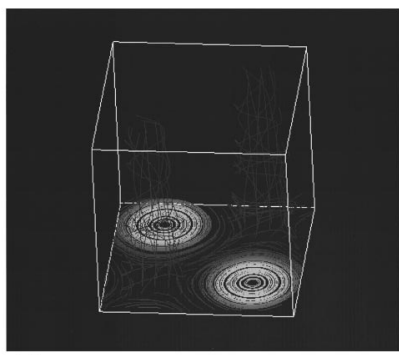

(d) intensified and actually even more strongly than $\gamma$.

Now we consider the three-dimensional structure associated with the three-dimensional enstrophy given by

FIG. 7. Vortex lines for the initial condition 1 ; (a) $t=0$, (b) $t=0.6$, (c) $t$ $=1.2$, and (d) $t=1.4$. 




FIG. 8. Time evolution of the norms for the initial condition $2 ; E_{\gamma}(t)$ (dashed) and $Q_{\gamma}(t)$ (solid).

there are many cells in $|\boldsymbol{\Omega}|^{2}$ initially, because of its quadratic nature. At later times, prominent cells appear in the two regions where $\gamma$ is strongly negative. As we have seen above, there the axial component $\omega$ is small. It is the azimuthal components of vorticity [parallel to the $(x, y)$ plane] that are stretched intensely, contributing to the large threedimensional enstrophy. It should also be noted that the enstrophy is not uniformly distributed inside the cells, but are highly concentrated in ring-like regions. Near the centers the enstrophy is very small. This feature is also seen by observing the shape of isosurfaces. The intense vorticity regions have a characteristic structure of semi-infinite cylinders associated with two-dimensionalization of the vorticity field, that is, hollowing vortices.

We show in Fig. 7 the vorticity lines which start from a plane $z=2 / 64 \times 2 \pi$ for the corresponding times. In the regions with negative $\gamma$, the vorticity lines lie on the plane, because the axial component of vorticity becomes very small due to strong compression onto the $(x, y)$ plane. On the other hand, in regions with positive $\gamma$ the vorticity lines make spirals by the nonzero axial component of vorticity. A more detailed analysis on the maximum $\max _{x}|\gamma(x, t)|$ is deferred to Sec. III.

\section{B. Initial condition 2}

We have seen that the amplification of $|\gamma|$ in its negative region is the key process leading to finite-time singularity. A question arises here is what kind of properties are required in the initial conditions of $\gamma$ for such singularities to form. To study this point, we employ initial conditions which have a single maximum in the fields. The first choice of this kind is

$$
\omega=\gamma=W=\frac{1}{c}\left(2-\left(\frac{r}{a}\right)^{2}\right) \exp \left(-\frac{1}{2}\left(\frac{r}{a}\right)^{2}\right),
$$

where $r=\sqrt{(x-\pi)^{2}+(y-\pi)^{2}}$. This initial condition is proportional to $\Delta \exp \left(-1 / 2(r / a)^{2}\right)$ and has a peak at $r=0$.

We use $a=0.3, c=0.2$ for constants. The constant $a$ is chosen to make $\omega, \gamma$, and $W$ sufficiently small, typically $O\left(10^{-16}\right)$, at the boundaries $r=0,2 \pi$ so that it does not conflict with periodic boundary conditions in practice. The normalization constant $c$ is chosen to make the norm of $\gamma$ not too small compared with the time increment.

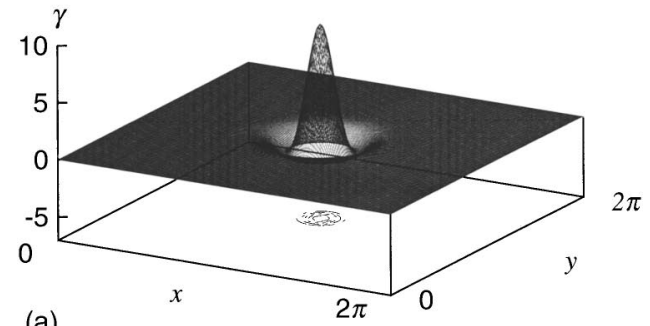

(a)

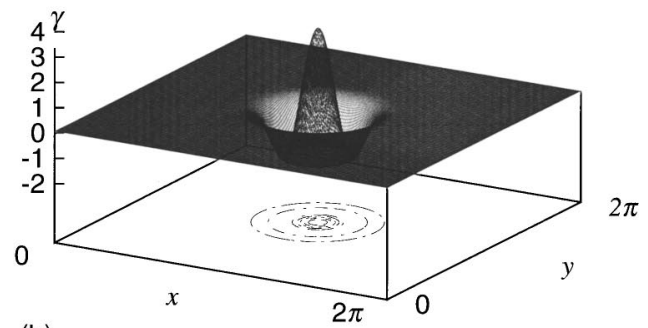

(b)

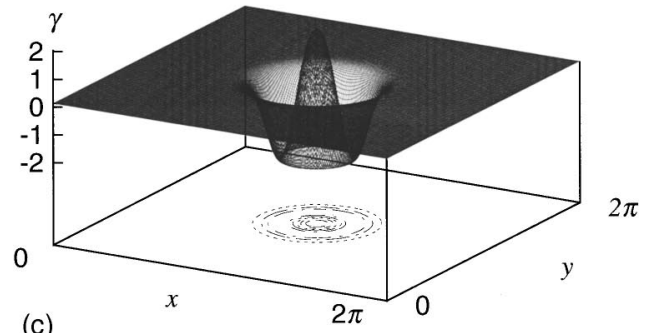

(c)

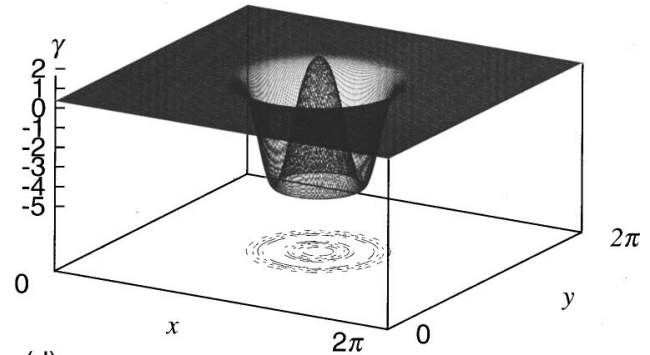

(d)

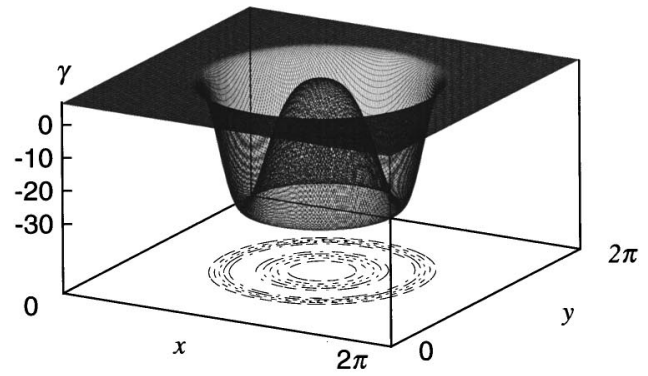

(e)

FIG. 9. Perspective plots and contours of $\gamma$ for the initial condition 2; (a) $t=0$, (b) $t=0.2$, (c) $t=0.4$, (d) $t=0.6$, and (e) $t=0.8$.

Here we take all the three fields identical so that initially the negative $\gamma$ part is not so strongly stressed. The minimum value of $\gamma$ is $\gamma(2 a)=-2 e^{-2} / c \approx-1.35$, so, on the rhs of (21) we have $-\gamma^{2} \approx-1.82$ and $2\left\langle\gamma^{2}\right\rangle \approx 0.8$. Therefore, at some points the rhs is mildly negative $\approx-1$, in comparison to the maximum of $\gamma(0)=5$.

We show the time evolution is $E_{\gamma}(t)$ and $Q_{\gamma}(t)$ in Fig. 

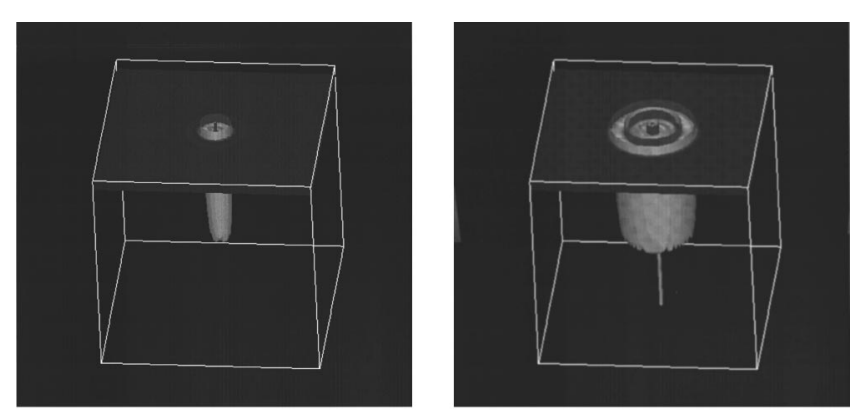

(a)



(c)

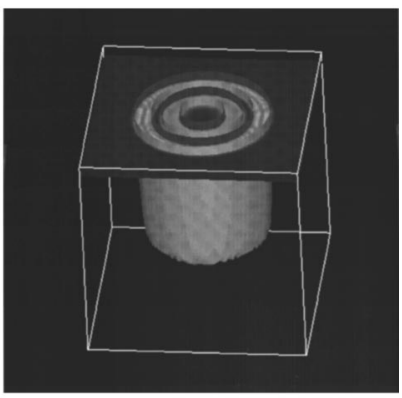

(d)

FIG. 10. Three-dimensional enstrophy for the initial condition 2 ; (a) $t=0$, (b) $t=0.4$, (c) $t=0.6$, and (d) $t=0.8$.

8. These norms of $\gamma$ decrease in the very early stage, but they start to increase later. They apparently blow up at around $t=0.83$. No difference has been found between computations performed with $256^{2}$ and $512^{2}$ grid points. We have checked that the Fourier spectra decay exponentially at large wave numbers, close to the late stage of computation (figures omitted).

In Fig. 9 the perspective plots of $\gamma$ are shown at $t$ $=0,0.2,0.4,0.6$, and 0.8 . The region with negative $\gamma$ has initially a small circle. As time elapses, the value of $\gamma$ at the ring-like region decreases in time, getting more and more negative. It should also be noted that the size of this ring is getting larger and larger, consistent with the interpretation that the underlying instability has its origin in the large-scale components. Because of symmetry imposed as an initial condition, the finally singular set has a line-like structure.

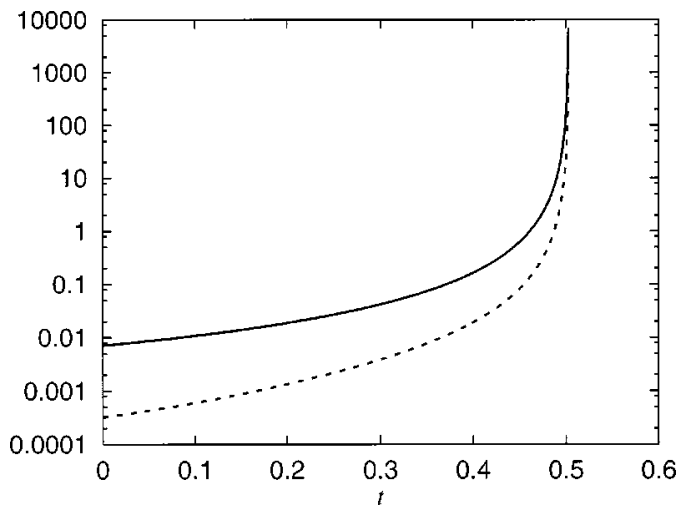

FIG. 11. Time evolution of the norms for the initial condition $3 ; E_{\gamma}(t)$ (dashed) and $Q_{\gamma}(t)$ (solid).

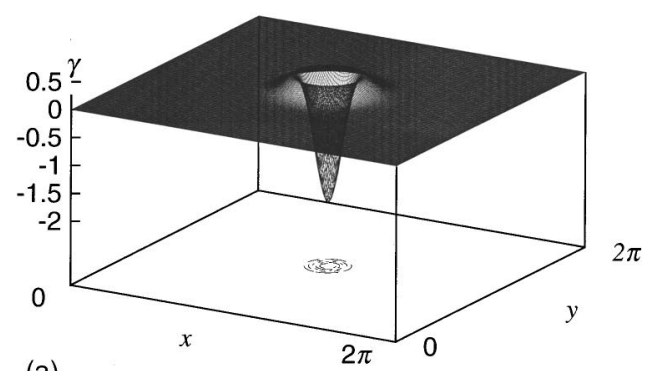

(a)

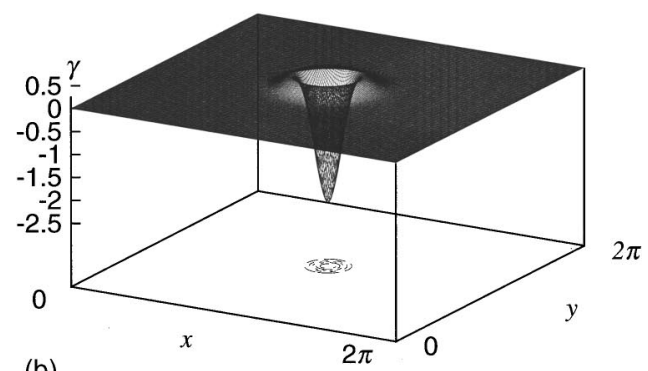

(b)
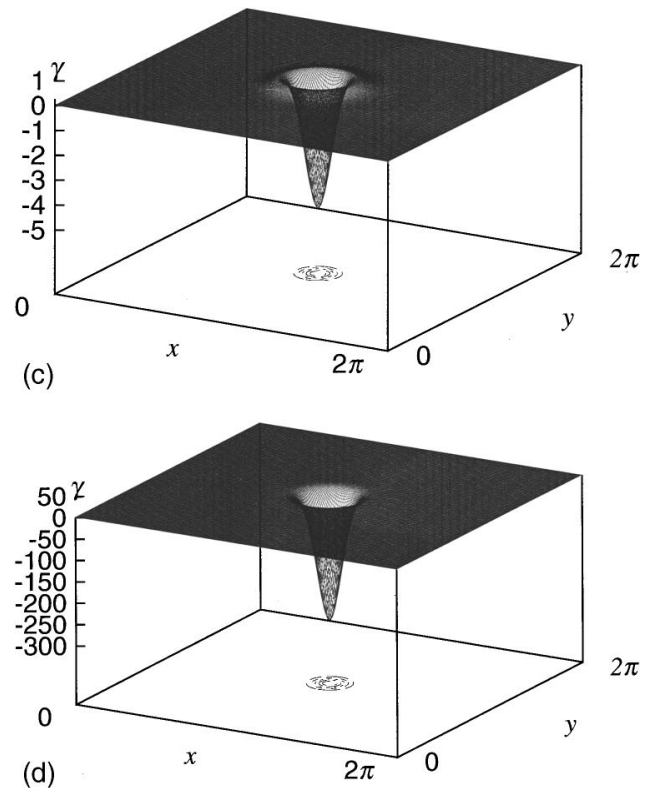

FIG. 12. Perspective plots and contours of $\gamma$ for the initial condition 3; (a) $t=0$, (b) $t=0.1$, (c) $t=0.3$, and (d) $t=0.5$.

We show the corresponding three-dimensional enstrophy distribution $|\boldsymbol{\Omega}|^{2}$ in Fig. 10. Isosurfaces of enstrophy density form two cylinders, inside which the enstrophy density is very high. These ring-like structures correspond to those of negative $\gamma$ in Fig. 9. They are also similar to the ones shown in Figs. 6(c) and 6(d).

\section{Initial condition 3}

As a final choice of initial condition, we reverse the signs of $\gamma$ and $W$ in (41) to consider

$$
\omega=-\gamma=-W=\frac{1}{c}\left(2-\left(\frac{r}{a}\right)^{2}\right) \exp \left(-\frac{1}{2}\left(\frac{r}{a}\right)^{2}\right) .
$$

This is more singular than (41) in that it stresses the negative part of $\gamma$. For the parameters we take $a=0.3, c=1$. We show time evolution of $E_{\gamma}(t)$ and $Q_{\gamma}(t)$ in Fig. 11. Unlike 


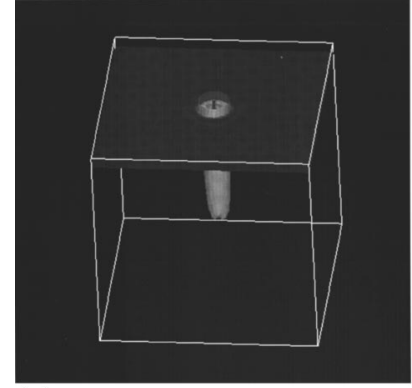

(a)



(b)

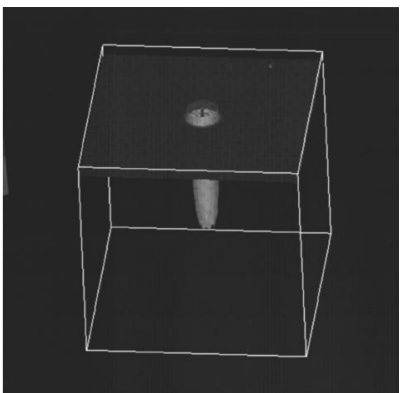

(c)

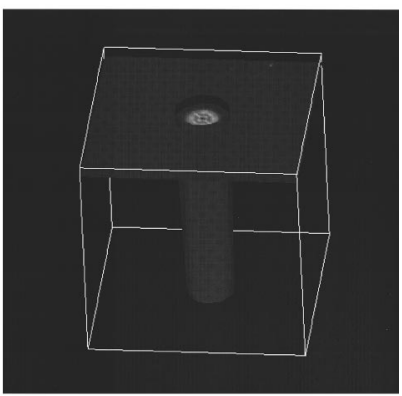

(d)

FIG. 13. Three-dimensional enstrophy for the initial condition 3; (a) $t=0$, (b) $t=0.1$, (c) $t=0.3$, and (d) $t=0.5$.

the initial condition 2, they start increasing right after $t=0$ and both of them appear to diverge at $t \approx 0.504$.

Perspective plots of $\gamma$ are shown in Fig. 12 at $t$ $=0,0.1,0.3,0.5$. The shape of $\gamma$ hardly changes in time but the negative peak in the initial condition of $\gamma$ keeps increasing in magnitude until the end of numerical calculation.

In Fig. 13 we also show three-dimensional enstrophy distribution at corresponding times. The pencil-like structure is observed at each time and its shape does not appear to change significantly. This suggests that if there is a strongly negative $\gamma$ in the initial condition, then the subsequent evolution is dominated by the presence of this negative peak.

\section{ALTERNATIVE APPROACH TO THE PROBLEM}

\section{A. Taylor series expansion in time}

In the previous section we have used pseudo-spectral computations to see whether numerical solutions of the Euler equations blow up in finite time. Here we study the formation of singularities using a different approach by tracing the evolution of norms with Taylor series expansions in time. This well known method has been applied in the past to the conventional Euler equations. ${ }^{12,24,25}$ However, as we shall see shortly, its application to the system of Eqs. (21)-(23) has some distinctive features which are absent in its application to the conventional problem. This method enables us to examine the apparent singularity extremely accurately.

Since $\gamma$ is the most important field, we pay special attention to its moments defined by:

$$
I_{p}(t)=\left\langle\gamma(\boldsymbol{x}, t)^{p}\right\rangle, \quad \text { for } p=0,1,2, \ldots
$$

It is straightforward to show that $I_{p}(t)$ satisfy the following set of infinite numbers of ordinary differential equations:

$$
\frac{d I_{p}}{d t}=-(p+1) I_{p+1}+2 p I_{2} I_{p-1}, \quad \text { for } p=0,1,2, \ldots,
$$

where we define $I_{-1}=0$. This set of equations is not closed, but introducing a double series $a_{p, n}$ as

$$
I_{p}=\sum_{n=0}^{\infty} a_{p, n} t^{n},
$$

we can derive the following recursion relationship for it:

$$
\begin{aligned}
a_{p, n+1}= & \frac{1}{n+1}\left(-(p+1) a_{p+1, n}+2 p \sum_{l=0}^{n} a_{2, l} a_{p-1, n-l}\right), \\
& \text { for } p \geqslant 0, n \geqslant 0 .
\end{aligned}
$$

The series $a_{p, n}$ should satisfy the following conditions:

$$
\begin{aligned}
& a_{0,0}=1, \\
& a_{0, n}=0, \quad \text { for } n=1,2,3, \ldots,
\end{aligned}
$$

and

$$
a_{1, n}=0, \quad \text { for } n=0,1,2,3, \ldots \text {. }
$$

The first two conditions are trivial, and the third one follows from the constraint $\langle\gamma\rangle=0$. Since the rhs of (46) is made up of coefficients $a_{m, n}$ whose index $m$ is less than $p$, we can solve for $a_{p, n}$ by iteration. In practice, we start generating $a_{p, 0}$ for $0 \leqslant p \leqslant N+2$ with a large $N$. For the specific choice of initial condition 1 , it is easy to show for the initial condition 1

$$
a_{p, 0}=\left\{\begin{array}{l}
\left(\frac{p !}{2^{(p / 2)}(p / 2) !}\right)^{2} \text { for even } p . \\
0 \text { for odd } p
\end{array}\right.
$$

Then, by setting $n=1,2,3 \ldots$ in (46), we obtain successively $a_{2, n}$ for $0 \leqslant n \leqslant N, a_{3, n}$ for $0 \leqslant n \leqslant N-1$, and in general $a_{p, n}$ for $0 \leqslant n \leqslant N+2-p$. This procedure has been done by symbolic manipulation using Maple $V$ for $N=500$. In this way we obtain the exact Taylor coefficients of, for example, $I_{2}(t)=\left\langle\gamma^{2}\right\rangle$ up to $O\left(t^{500}\right)$.

It should be noted that such a high-order can be achieved, because in Eq. (21) for $\gamma$ the pressure term does not appear explicitly. Recall that in the case of usual Euler equation where the Hessian matrix of pressure term couples with vorticity and rate-of-strain, the above reduction to double series is not possible.

Using the Taylor coefficients thus rigorously obtained, we examine the analytic structure of the moments $I_{p}(t)$. They turned out to be even (odd) functions of time $t$ for even (odd) $p$. Defining $\xi=t^{2}$ we write

$$
I_{p}=\sum_{n=0}^{\infty} a_{p, 2 n} \xi^{n},
$$

for even $p$ and

$$
I_{p}=t \sum_{n=0}^{\infty} a_{p, 2 n+1} \xi^{n},
$$

for odd $p$. 


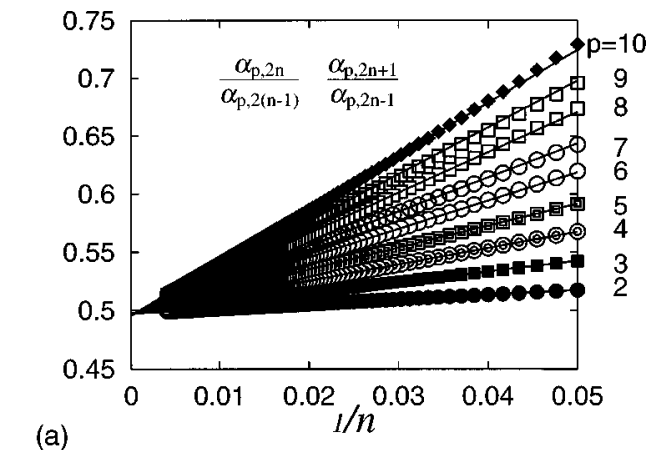

(a)
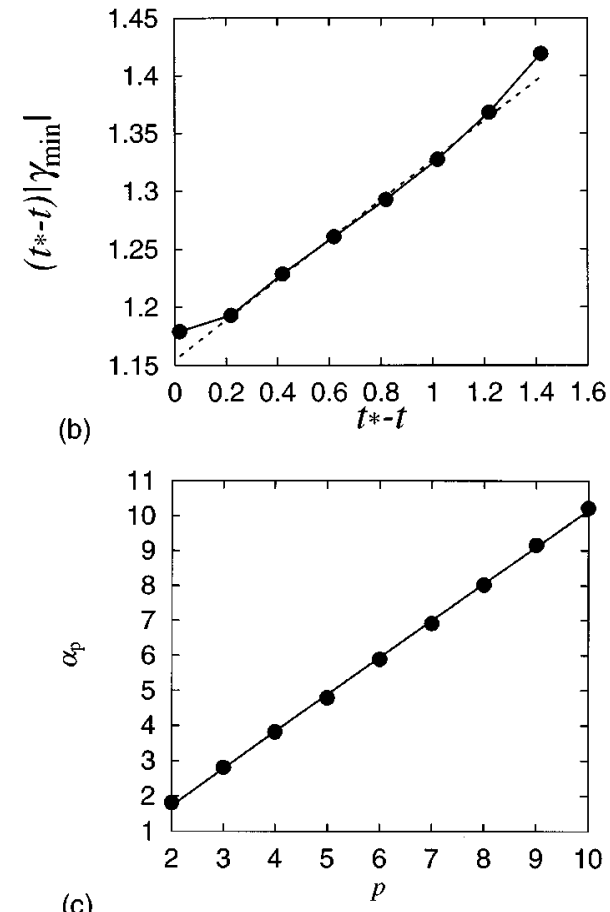

(c)

FIG. 14. Detailed analyses of the case of the initial condition 1. (a) The Domb-Sykes plot for $p=2,3, \ldots 10$ together with fitted straight lines. (b) The time evolution of $\left(t_{*}-t\right)\left|\gamma_{\min }\right|$ against $t_{*}-t$ together with a fitted straight line (dashed). (c) The exponents $\alpha_{p}$, together with a fitted straight line.

\section{B. The Domb-Sykes plot}

Here we examine whether or not the Taylor-series expansion analysis is consistent with the pseudo-spectral computations described in Sec. II and some mathematical analysis.

We try the usual Domb-Sykes plots by fitting

$$
\frac{a_{p, 2 n}}{a_{p, 2(n-1)}} \approx \frac{1}{\rho_{p}}\left(1+\frac{\alpha_{p}-1}{n}\right) \quad \text { as } n \rightarrow \infty
$$

and

$$
\frac{a_{p, 2 n+1}}{a_{p, 2 n-1}} \approx \frac{1}{\rho_{p}}\left(1+\frac{\alpha_{p}-1}{n}\right) \quad \text { as } n \rightarrow \infty,
$$

respectively, for even and odd $p$. Here $\rho_{p}$ is the radius of convergence and $\alpha_{p}$ is the strength of the singularity such that $I_{p} \propto\left(\rho_{p}-\xi\right)^{-\alpha_{p}}$ as $\xi \rightarrow \rho_{p}$.

In Fig. 14(a), we plot the ratios $a_{p, 2 n} / a_{p, 2(n-1)}$ against $1 / n \quad$ for $\quad p=2,4, \ldots, 10, \quad n=1,2, \ldots(N+2-p) / 2 \quad$ and $\left(a_{p, 2 n+1}\right) /\left(a_{p, 2 n-1}\right) \quad$ against $1 / n$ for $p=3,5, \ldots, 9, \quad n$
TABLE I. Fitted values of $\alpha_{p}$ and $\rho_{p}$ in the Domb-Sykes plot.

\begin{tabular}{rcr}
\hline \hline$p$ & $\alpha_{p}$ & $\rho_{p}$ \\
\hline 2 & 0.49748702431861 & 1.8118243673555 \\
3 & 0.49748366006028 & 2.8125811671362 \\
4 & 0.49736430984726 & 3.8218680292620 \\
5 & 0.49737625333089 & 4.7890517385808 \\
6 & 0.49703631941514 & 5.8911039911952 \\
7 & 0.49673791157762 & 6.9143197712255 \\
8 & 0.49652364387792 & 8.0237105414669 \\
9 & 0.49566993397728 & 9.1619462335132 \\
10 & 0.49581409456195 & 10.2271058579109 \\
\hline \hline
\end{tabular}

$=1,2, \ldots(N+1-p) / 2$. Clear straight lines are observed at large $n$. By using the least squares fitting in $n \geqslant 20$, we determined $\alpha_{p}$ and $\rho_{p}$ for $p=2,3, \ldots, 10$ numerically (see Table I). More precisely, the fitting procedure was performed after converting the exact ratios into floating point real numbers in double precision arithmetic. The results do not depend significantly on the choice of interval used for fitting. It should be noted that the nearly same value are consistently obtained for the radii of convergence at different orders.

For example, we find that $1 / \rho_{2} \approx 0.497$ and $\alpha_{2} \approx 1.8$, which corresponds to a blow-up of the form

$$
\left\langle\gamma^{2}\right\rangle \propto \frac{1}{\left(t_{*}-t\right)^{\alpha_{2}}},
$$

at $t_{*}=\sqrt{\rho_{2}} \approx 1.42$. (Note that $\xi=2$ corresponds to $t=\sqrt{2}$.) This is close to the time $t=1.419$ at which the pseudospectral computation breaks down. It should be noted that the two completely different analyses, pseudo-spectral computation and Taylor series expansion, give essentially the same answer for the time of blow-up. This suggests the blow-up found in the numerics is a real one rather than a numerical artifact. This observation has fostered mathematical analyses of the equations. As one of mathematical results we have the following criterion similar to BKM criterion; ${ }^{3}$ if a smooth solution breaks down in finite time $t_{*}$ then we must have

$$
\lim _{t \rightarrow t_{*}} \int_{0}^{t} \max |\gamma(\boldsymbol{x}, s)| d s=\infty .
$$

This constrains, as usual, the exponent of possible algebraic blow-up as

$$
\max _{x}|\gamma(x, t)| \propto \frac{1}{\left(t_{*}-t\right)^{\beta}}, \quad(\beta \geqslant 1) .
$$

In Fig. 14(b) we show $\left(t_{*}-t\right) \max _{x}|\gamma(x, t)|$ against $t_{*}-t$, where we take $t_{*}=1.419$. The straight line suggests that the blow-up occurs at the marginal exponent $\beta=1$. Then we try to fit it in the form

$$
\max _{x}|\gamma(\boldsymbol{x}, t)|=\frac{a}{t_{*}-t}+b,
$$

by the least-squares fitting using data $t=0.2,0.4, \ldots, 1.2$. The values obtained are $a=1.155$ and $b=0.172$.

In this numerical simulation, not only the local quantity $\max _{x}|\gamma(\boldsymbol{x}, t)|$ but also the global one $\left\langle\gamma(\boldsymbol{x}, t)^{2}\right\rangle$ blow up si- 
TABLE II. Poles of the Padé approximants.

\begin{tabular}{|c|c|}
\hline Approximants & Poles in $\xi$ \\
\hline$[5,5]$ & $\begin{array}{l}-9.751524732, \quad 0.00006012485040, \quad 1.950075790 \text {, } \\
\quad 2.170674366,4.358095782\end{array}$ \\
\hline$[10,10]$ & $\begin{array}{l}-13.97014338,-11.82050188,0.1029677908 \times 10^{-9}, \\
\quad 1.999349184,2.035354536, \\
\text { 2.264 591266, 2.745170597, 3.742644283, } \\
\quad 6.228639034,23.69527562\end{array}$ \\
\hline$[15,15]$ & $\begin{array}{l}-84.05640948,-18.40248970,-12.84562099 \\
-12.14978880,0.2415944078 \times 10^{-15} \\
\quad 2.006210486,2.019948936,2.107673513 \\
\quad 2.267992708,2.526846629 \\
\quad 2.931388994,3.575127401,4.663375978 \\
\quad 6.735971424,11.77735994\end{array}$ \\
\hline$[20,20]$ & $\begin{array}{l}-52.71775405,-20.94615282,-14.81984005, \\
-12.58283142,-12.24822016, \\
0.6280191510 \times 10^{-21}, 2.008345498, \\
2.015440843, \quad 2.061734822,2.143166740, \\
2.266556903,2.441942930,2.685359351, \\
3.022650991,3.496884366, \\
4.183872970,5.228015126,6.936378207, \\
10.07036306,16.92990022\end{array}$ \\
\hline$[25,25]$ & $\begin{array}{l}-60.78482275,-23.96513020,-16.75640947, \\
-13.81052723,-12.49080586, \\
-12.28606612,0.2672783928 \times 10^{-26}, 2.009251038, \\
\quad 2.013592533,2.042501762, \\
\quad 2.092591393,2.166619817,2.268134969, \\
\quad 2.402372954,2.576940742, \\
\quad 2.802920690,3.096732506,3.483402514, \\
\quad 4.002621749,4.720779646, \\
\quad 5.757112164,7.347644789,10.02817942, \\
15.25142079,24.46214386\end{array}$ \\
\hline
\end{tabular}

multaneously in finite time. In order for this observation to be consistent with the rhs of (21), it is necessary that $\alpha_{2}$ $\leqslant 2$. As we have seen above, this is actually satisfied.

To characterize the singular structure in $\gamma$ we consider higher-order exponents $\alpha_{p}$. In Fig. 14(c) we plot the exponents $\alpha_{p}$ against $p$ for $p=2,3, \ldots, 10$ which displays a clear linear behavior in $p$. A least-squares fitting was used to give

$$
\alpha_{p}=c_{1} p+c_{2},
$$

where $c_{1}=1.054$ and $c_{2}=-0.385$. Noting that

$$
\max _{x}|\gamma(\boldsymbol{x}, t)|=\lim _{p \rightarrow \infty}\left(I_{p}\right)^{\frac{1}{p}} \lim _{p \rightarrow \infty} \frac{1}{\left(\rho_{p}-\xi\right)^{c_{1}+c_{2} / p}},
$$

and that $\rho_{p}$ is independent of $p$, we find a singular behavior of the form

$$
\max _{x}|\gamma(\boldsymbol{x}, t)| \propto \frac{1}{\left(t_{*}-t\right)^{c_{1}}} .
$$

This is consistent with (57) because $c_{1}>1$. Moreover it should be noted that $c_{1}$ is close to unity. These constants $c_{1}$ and $c_{2}$ are obtained from $a_{p, n}$ using $n \geqslant 20$. If we fit $\alpha_{p}$ from $a_{p, n}$ using $n \geqslant 50$, we obtain $c_{1}=1.027, c_{2}=-0.281 ; c_{1}$ is obtained even closer to unity. This suggests a possibility that $c_{1}$ is exactly equal to unity, which is consistent with (58). While we cannot conclude that $c_{1}=1$ because of the limited

\begin{tabular}{|c|c|}
\hline Approximants & Zeros in $\xi$ \\
\hline$[5,5]$ & $\begin{array}{l}-2.216943412,0.00006012485041,3.271947112, \\
16.13758183-2.582369765 \mathrm{i}, \\
16.13758183+2.582369765 \mathrm{i}\end{array}$ \\
\hline$[10,10]$ & $\begin{array}{l}-30.19467096,-2.217048815,0.1029677908 \times 10^{-9}, \\
2.203407075,2.593186669,3.362981535, \\
5.037640032,10.14553384, \\
18.28975406-25.90834767 \mathrm{i}, \\
18.28975406+25.90834767 \mathrm{i}\end{array}$ \\
\hline$[15,15]$ & $\begin{array}{l}-32.58058020,-16.14103113, \mathrm{n}-2.217048815, \\
0.2415944078 \times 10^{-15}, 2.088216892, \\
2.228341745,2.432709408-56.61758878 \mathrm{i}, \\
2.432709408+56.61758878 \mathrm{i} \\
\quad 2.455370758,2.806344773,3.351514526 \\
\quad 4.234610167,5.791686158 \\
\quad 9.009345310,18.29747596\end{array}$ \\
\hline$[20,20]$ & $\begin{array}{l}-32.44397406,-18.44184439,-14.11757089 \text {, } \\
-4.388434788-72.06414749 \mathrm{i}, \\
-4.388434788+72.06414749 \mathrm{i},-2.217048814, \\
0.6280191510 \times 10^{-21}, 2.052487862, \\
2.125475460,2.237354559,2.396706302, \\
2.617040931,2.919720128, \\
3.339398039,3.934872487,4.812575562, \\
6.183157666,8.517715024, \\
13.04895794,23.19869932\end{array}$ \\
\hline$[25,25]$ & $\begin{array}{l}-36.66290657,-20.90397806,-15.76540294, \\
-13.44484493, \\
-2.422275806-72.03562073 \mathrm{i}, \\
-2.422275806+72.03562073 \mathrm{i},-2.217048815, \\
0.2672783928 \times 10^{-26}, 2.037117960,2.082587744, \\
2.150718578,2.244666576, \\
2.369045838,2.530502054,2.738607574, \\
3.007333373,3.357561888, \\
3.821584980,4.451655754,5.337545534, \\
6.646338905,8.725034081, \\
12.40843322,19.72399657,27.18119371\end{array}$ \\
\hline
\end{tabular}
maximum order of $I_{p}$, the Taylor series analysis in time is
TABLE III. Zeros of the Padé approximants.

consistent with not only the pseudo-spectral computations but also mathematical results in every aspect we have checked.

\section{Padé approximation}

In order to further strengthen the result on the presence of blow up, we apply the Padé approximation to the $I_{2}(t)$. We determine $[M, L]$-Padé approximants of $I_{2}$ by representing it as a rational function of $\xi=t^{2}$, whose degree of denominator is $M$ and that of numerator is $L$. Up to this point, the computation is exact. In Table II, we document the location of poles of approximants, that is, roots of the denominator which are obtained by solving the algebraic equations numerically. We also show in Table III the location of zeros of approximants, that is, roots of the numerator in a similar manner. All the poles are found to be real in $\xi$ but some of the zeros come out in conjugate pairs of complex numbers.

There are poles on the positive real $\xi$ axis, whose distance to the origin is very small. For example, the $[10,10]$-approximant has a pole at $0.1029677908 \times 10^{-9}$. However, exactly at this location there is a zero, which compensates the singular behavior of the apparent pole. ${ }^{26}$ This is consistent because there should not be singular behavior 


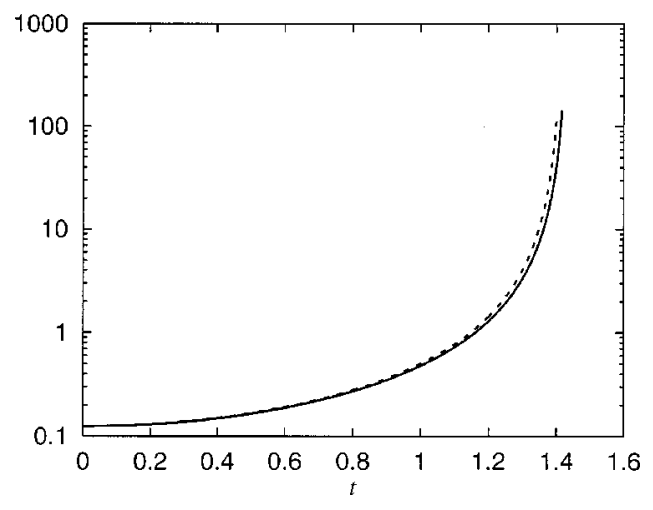

FIG. 15. Time evolution of $Q_{\gamma}(t)$ for the initial condition 1, the NavierStokes case (solid) and the Euler case (dashed).

close to the initial condition. Discarding these poles, the first relevant positive poles are located at $\xi=1.999349184$ for the $[10,10]$-approximant. Checking the location of zeros in Table III, we see that the pole is not compensated by a zero. We note that these results are irrelevant to the choice of orders of approximants. Thus the analysis by Padé approximation also supports the presence of a real-time singularity near $\xi=2$.

\section{THE VISCOUS PROBLEM}

In this section, we compare the Euler solution with a corresponding Navier-Stokes solution. The main objective is to see whether or not the singularity formation found in the inviscid case is prevented by a small amount of viscosity.

The Navier-Stokes version of the class of solutions under consideration satisfy the following set of equations: ${ }^{15}$

$$
\begin{aligned}
& \frac{\partial \omega}{\partial t}+(\boldsymbol{u} \cdot \nabla) \omega=\gamma \omega+\nu \triangle \omega, \\
& \frac{\partial \gamma}{\partial t}+(\boldsymbol{u} \cdot \nabla) \gamma=-\gamma^{2}+2\left\langle\gamma^{2}\right\rangle+\nu \triangle \gamma,
\end{aligned}
$$

and

$$
\frac{\partial W}{\partial t}+(\boldsymbol{u} \cdot \nabla) W=-\gamma W+\nu \triangle W .
$$

The condition $\langle\gamma\rangle=0$ must also be satisfied.

We consider the initial condition 1 used in Sec. III A with $\nu=0.005$. We compare the time evolution of the norm $Q_{\gamma}(t)$ between Navier-Stokes and Euler equations in Fig. 15 . The growth of the norm is only slightly suppressed by the effect of viscosity, but it still appears to diverge approximately at $t=1.433$, which is slightly later than the time of blow-up for the inviscid case. The contribution coming from the viscous terms works to suppress the growth of the quantity, as can be verified by the negative-definiteness of the last term of the following equation

$$
\frac{d Q_{\gamma}}{d t}=-\frac{3}{2}\left\langle\gamma^{3}\right\rangle-\nu\left\langle|\nabla \gamma|^{2}\right\rangle .
$$

Indeed the viscous term of (65) slows down the growth of $Q_{\gamma}(t)$ but actually is not so effective to prevent the singular- ity formation. This is consistent with the observation made in Fig. 2 that the instability come from the lower wave number part.

\section{SUMMARY AND DISCUSSION}

We have investigated numerically the class of solutions of Euler and Navier-Stokes equations identified in Ref. 15. We found that for a wide class of initial conditions the smooth Euler solutions appear to breakdown in finite time. At this time the ansatz (2) for the $3 \mathrm{D}$ velocity field is no longer valid and such a solution is no longer sustainable.

As one of the structural changes which can occur to vortex flows, it is of interest to compare the singular behavior observed here to the phenomenon of vortex breakdown. The latter phenomenon is characterized by "the formation of an internal stagnation point on the vortex axis, followed by reversed flow in a region of limited axial extent." ${ }^{, 27}$ Indeed, both are related with formation of a columnar vortex. In the case of vortex breakdown, the flow lying upstream the breakdown region is called the approach flow. This flow may be typically represented by the Burgers vortex, which is a special case of the class of solutions considered in this paper.

The breakdown region is associated with abrupt changes in the direction of axial velocity. This part can be well represented by a stationary smooth solution of the NavierStokes equations, see for example, Refs. 28 and 29. The term "breakdown" refers to a reversal of axial velocity but has nothing to do with mathematical breakdown of solutions. Downstream of the breakdown region a new vortex structure is formed, where the variation along the axis is gradual.

Some more differences should be noted. As noted in Ref. 29, the key process in vortex breakdown is the production of a negative azimuthal vorticity. But in our case, the axial vorticity tends to vanish in the regions where $\gamma$ blows down. Moreover, in the present case because the axial velocity is dominant over planar components, the streamlines basically lie on the cylindrical surfaces in the $z$ direction (figures omitted), in contrast to swirling streamlines of the breakdown region. In view of these differences and similarities, it may be appropriate to categorize the hollowing vortices documented here as one of the typical behaviors of the vortex flow.

Furthermore, there has been some work on vortex breakdown of a different type over the last few years. One is based on the flow in a channel with one end as a rotating disc; the problem is not the same as ours because a lot of stress is laid by authors on the boundary layer on the disc whereas we have an infinite channel. ${ }^{30,31}$ We also note that similar solutions of the Navier-Stokes equations have been investigated. ${ }^{32}$ There, the problem has been reduced to one dimension and a connection between the blow-up and the role played by convection term has been studied in detail.

Finally we mention some of the open problems regarding the class of solutions. First, we have not studied stability properties of the apparent singular solutions. We do not know whether the singular behavior found here may be unstable to small perturbations. Second, apparently there are multiple length scales involved in the formation of singulari- 
ties in the present problem. It may be interesting to seek for quasi-self-similar solutions. These are left for future investigations.

As mentioned in the Introduction, this class of solutions possesses unbounded velocity field only in one direction. We hope that this study may be regarded as an intermediate step toward understanding possible singularity formation of physically more interesting flows with better localization.

Note added in proof: According to a recent study ${ }^{33}$ by $\mathrm{P}$. Constantin, the blowup observed in this system can be proved, both for positive and negative $\gamma$. In this paper we paid little attention to the positive $\gamma$, since its behavior was not so prominent. However, a closer examination reveals that $\max \gamma$ also appears to diverge at the same time. Our computations are consistent with his analysis. Positive blow-up of $\gamma$ also means that the axial vorticity $\omega$ will also diverge [see Eq. (17)], as well as the azimuthal components that are driven by negative blow-up of $\gamma$.

\section{ACKNOWLEDGMENTS}

This work was done while the authors were attending a research program Turbulence, held during January 4-July 2, 1999, at the Isaac Newton Institute for Mathematical Sciences, University of Cambridge. A major part of computations were carried out on workstations there. We thank the Isaac Newton Institute for providing us with an excellent environment and pleasant hospitality. We also thank J. Brasseur, P. Constantin, Y. Couder, C. Doering, B. Galanti, F. Hussain, R. M. Kerr, T. S. Lundgren, S. Malham, H. K. Moffatt, H. Okamoto, and J. C. Vassilicos for helpful discussion. We are also grateful to an anonymous referee for suggesting useful comments.

${ }^{1}$ R. M. Kerr, "Evidence for a singularity of the three-dimensional, incompressible Euler equations,' Phys. Fluids A 5, 1725 (1993).

${ }^{2}$ J. Leray, "Sur le mouvement d'un liquide visqueux emplissant l'espace," Acta Math. 63, 193 (1934).

${ }^{3}$ J. T. Beale, T. Kato, and A. Majda, "Remarks on the breakdown of smooth solutions for the 3D Euler equations," Commun. Math. Phys. 94, 61 (1984).

${ }^{4}$ P. Constantin, Ch. Fefferman, and A. Majda, "Geometric constraints on potentially singular solutions for the 3D Euler equations," Comm. PDEs 21, 559 (1996).

${ }^{5}$ P. Constantin, "Geometric statistics in turbulence," SIAM Rev. 36, 73 (1994).

${ }^{6}$ G. Ponce, "Remarks on a paper by T. Beale, T. Kato and A. Majda," Commun. Math. Phys. 98, 349 (1985).

${ }^{7}$ S. Kida, "Three-dimensional periodic flows with high symmetry," J. Phys. Soc. Jpn. 54, 2132 (1985).

${ }^{8}$ R. Grauer and T. Sideris, "Numerical computation of three-dimensional incompressible ideal fluids with swirl," Phys. Rev. Lett. 67, 3511 (1991).

${ }^{9}$ O. N. Boratav and R. B. Pelz, "Direct numerical simulation of transition to turbulence from a high-symmetry initial condition," Phys. Fluids 6, 2757 (1994).

${ }^{10}$ A. Bhattacharjee, C. S. Ng, and X. Wang, "Finite time vortex singularity and Kolmogorov spectrum in a symmetric three-dimensional model," Phys. Rev. E 52, 5110 (1995).

${ }^{11} \mathrm{~A}$. Bhattacharjee and X. Wang, "Finite time vortex singularity and Kolmogorov spectrum in a symmetric three-dimensional model," Phys. Rev. Lett. 69, 2196 (1992).

${ }^{12}$ R. B. Pelz and Y. Gulak, "Evidence for a real-time singularity in hydrodynamics from time series analysis," Phys. Rev. Lett. 79, 4998 (1997).

${ }^{13}$ J. M. Burgers, "A mathematical model illustrating the theory of turbulence," Adv. Appl. Mech. 1, 171 (1948).

${ }^{14}$ H. K. Moffatt, S. Kida, and K. Ohkitani, "Stretched vortices-the sinews of turbulence; large-Reynolds-number asymptotics," J. Fluid Mech. 259, 241 (1994).

${ }^{15}$ J. D. Gibbon, A. Fokas, and C. R. Doering, "Dynamically stretched vortices as solutions of the Navier-Stokes equations," Physica D 132, 497 (1999).

${ }^{16}$ J. T. Stuart, "Nonlinear Euler partial differential equations: Singularities in their solution," in Proceedings in Honor of C. C. Lin, edited by D. J. Benney, C. Yuan, and F. H. Shu (World Scientific, Singapore, 1987), pp. 81-95.

${ }^{17}$ J. T. Stuart, "The Lagrangian picture of fluid motion and its implication for flow structures," IMA J. Appl. Math. 46, 147 (1991).

${ }^{18}$ S. J. Cowley, L. L. van Dommelen, and S. T. Lam, "On the use of Lagrangian variables in descriptions of unsteady boundary-layer separation," Philos. Trans. R. Soc. Lond., Ser. A 333, 343 (1990).

${ }^{19}$ S. Childress, G. R. Ierly, E. A. Spiegel, and W. R. Young, "Blow-up of unsteady two-dimensional Euler and Navier-Stokes solutions having stagnation point form,'” J. Fluid Mech. 203, 1 (1989).

${ }^{20} \mathrm{P}$. Constantin, "Note on the loss of regularity for solutions of the $3 \mathrm{D}$ incompressible Euler and related equations," Comm. Math. Phys. 104, 311 (1986).

${ }^{21} \mathrm{H}$. K. Moffatt, "The interaction of skewed vortex pairs: A model for blow-up of the Navier-Stokes equations," J. Fluid Mech. 409, 51 (2000).

${ }^{22} \mathrm{C}$. W. Oseen, "Exakte Lösungen der hydrodynamischen Differentialgleichungen. II," Ark. Mat., Astronom. Fys. 20A, 1 (1927).

${ }^{23}$ T. Lundgren, "Strained spiral vortex model for turbulent fine structure," Phys. Fluids 25, 2193 (1982).

${ }^{24}$ M. E. Brachet, D. I. Meiron, S. A. Orszag, B. G. Nickel, R. H. Morf, and U. Frisch, "Small-scale structure of the Taylor-Green vortex,', J. Fluid Mech. 130, 411 (1983).

${ }^{25}$ R. H. Morf, S. A. Orszag, and U. Frisch, "Spontaneous singularity in three-dimensional inviscid incompressible flow," Phys. Rev. Lett. 44, 572 (1980).

${ }^{26}$ This phenomenon is known as "defects." See, for example, G. A. Baker and P. G.-Morris, Padé Approximants, 2nd ed., Encyclopedia of Mathematics and its Applications, Vol. 59 (Cambridge University Press, Cambridge, England, 1996).

${ }^{27}$ S. Liebovich, "The structure of vortex breakdown," Annu. Rev. Fluid Mech. 10, 221 (1978).

${ }^{28}$ J. M. Lopez, “Axisymmetric vortex breakdown Part 1: Confined swirling flow," J. Fluid Mech. 221, 533 (1990).

${ }^{29}$ G. L. Brown and J. M. Lopez, “Axisymmetric vortex breakdown Part 2: Physical mechanisms,", J. Fluid Mech. 221, 553 (1990).

${ }^{30}$ K. Stewartson, C. J. Simpson, and R. J. Bodonyi, “The unsteady boundary layer on a rotating disk in a counter rotating fluid. Part 2,' J. Fluid Mech. 121, 507 (1982).

${ }^{31}$ P. Hall, P. Balakumar, and D. Papageorgiu, "On a class of unsteady three-dimensional Navier-Stokes solutions relevant to rotating disc flows: Threshold amplitudes and finite-time singularities," J. Fluid Mech. 238, 297 (1992).

${ }^{32} \mathrm{H}$. Okamoto and J. Zhu, "Some similarity solutions of the Navier-Stokes equations and related topics," Taiwanese J. of Math. 4, 65 (2000).

${ }^{33} \mathrm{P}$. Constantin, "The Euler equations and non-local conservative Riccati equations," Internat. Math. Res. Notices (IMRN) 9, 455 (2000). 